Extinctions: \section{conserve not collate}

Fangliang He and Stephen Hubbell correct an overestimation of $160 \%$ for species extinction rates resulting from habitat destruction (Nature 473, 368-371; 2011). However, near-term extinction rates predicted by the Millennium Ecosystem Assessment still remain at 400-4,000 times the background rate of species extinction.

Although it may help to refine future predictions, we caution against their recommendation for collating more detailed geographical data as an urgent priority for conservation science.

Knowing where species occur and their risk of extinction is fundamental for deciding where to focus efforts to protect them. But the diminishing returns on the value of biological surveys (H. S. Grantham et al. Conserv. Lett.1, 190-198; 2008) means that more data may not translate into significantly better decisions. Heterogeneity in the costs and likelihood of success of conservation actions can influence investment priorities far more.

Areas designated a priority for species protection, identified using the 'speciesarea relationship', are not affected by model uncertainty, taxonomic group or the nonrandom distribution of species (M. C. Evans et al. Divers. Distrib. 17, 437-450; 2011).

Megan Evans, Hugh Possingham, Kerrie Wilson The University of Queensland, Australia.m.evans1@uq.edu.au

\section{Extinctions: consider all species}

We question Fangliang He and Stephen Hubbell's claim that species-area relationships overestimate global extinction

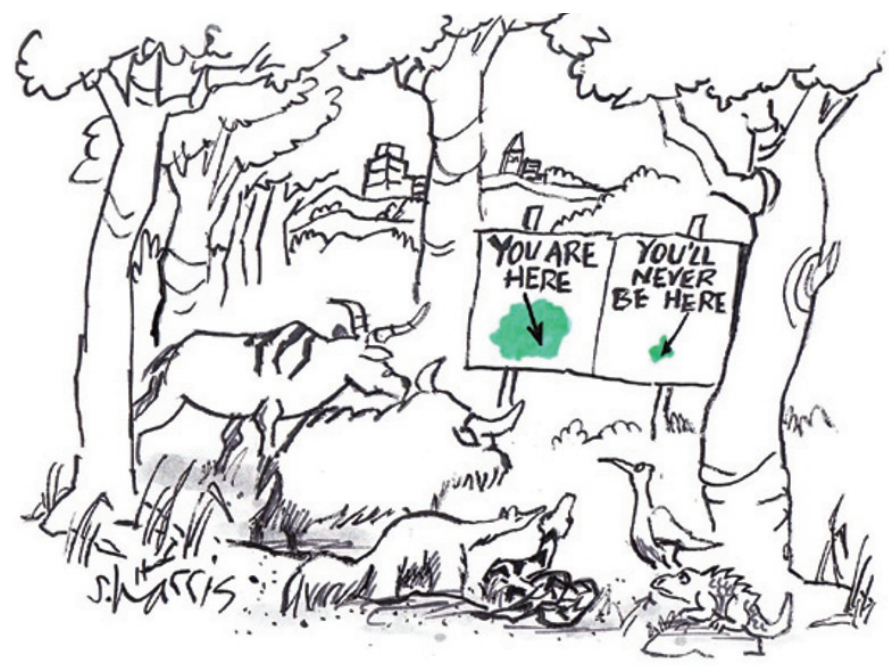

(Nature 473, 368-371; 2011). We contend that they do not test their claims against real data on global extinction or threat. We also believe that they address only a small part of the problem.

Imagine destruction that wipes out $95 \%$ of habitat overnight - metaphorically speaking. How many species will have disappeared the following morning? He and Hubbell tell us it would be just those living only in the destroyed area, and not in the other $5 \%$. In our view, the more important question is how many species in total, including those in the remnant habitat 'islands' (the 5\%), will eventually become extinct (see M. L. Rosenzweig Species Diversity in Space and Time Cambridge Univ. Press, 1995.)

Many studies accurately verify extinction predictions based on the relationship between island area and numbers of species, which He and Hubbell dismiss. Scores of separate tests find striking agreement between the number of predicted extinctions from habitat loss and the number of consequent extinctions (or of species facing extinction). This is seen globally and within individual regions, including eastern North America, South America, Africa and southeast Asia (see, for example, S. L. Pimm and R. A. Askins Proc. Natl Acad.
Sci. USA 92, 9343-9347; 1995).

Comprehensive analyses can now combine remotely sensed ecosystem changes with information on species extinction risk, distribution, habitats, threats and conservation actions from the International Union for Conservation of Nature Red List. In our opinion, it is these studies - which ask the right questions and verify the answers - that have crucial implications for the world's efforts to conserve biodiversity.

T. M. Brooks ${ }^{\star}$ NatureServe, Virginia, USA.

tbrooks@natureserve.org

* On behalf of 7 co-signatories

(see go.nature.com/tsnlzs).

\section{Making society more resilient}

Japan's government would do well to consider how society can adapt to cope with the uncertainty and change caused by sudden disastrous natural events - called resilience thinking - rather than simply trying to overcome and eliminate such changes.

Catastrophic disturbances such as tsunamis, wildfires, flooding and volcanic eruptions can exact a huge human cost. But they may also have a positive impact on ecosystems, particularly those eroded by human activity. The 2004 Indian Ocean tsunami, for example, restored the beach nesting habitats for several threatened sea-turtle species (D. B. Lindenmayer and C. R. Tambiah Conserv. Biol. 19, 991; 2005).

The ability of ecosystems to absorb natural disturbances and society's ability to resist and recover from them are connected. History shows that socio-ecological systems that are resilient to hazards are less devastated by recurring natural events such as hurricanes (W. N. Adger et al. Science 309, 1036-1039; 2005). Ignoring the connection could lead to more unforeseen economic disasters. Akira S. Mori Yokohama National University, Japan. akkym@kb3.so-net.ne.jp

\section{Population decline is a long way off}

Fred Pearce uses strong words to criticize the United Nations' latest projected global population figures (Nature 473, 125; 2011).

But the UN's projections of a continuing rise in the population (see go.nature.com/wj3br5) are in line with its previous projections and with those of other major sources, including the US Census Bureau (see go.nature.com/ owcela) and the International Institute for Applied Systems Analysis (go.nature.com/cbg34l).

The new UN 'medium variant' projection expects 10.1 billion people by 2100,3 billion more than now. This is a sobering prospect for those concerned with human and environmental poverty.

In his book The Coming Population Crash (Beacon Press, 2010), Pearce predicts a drastic population decline owing to falling fertility. But the birth rate worldwide still exceeds the replacement rate, so the young greatly outnumber the old. The number of young women coming 